\title{
IMPACT and AIDIT: Strengthening Research Ties in Eastern Europe
}

\author{
Sarah Morgan', Jan Lubinski', Esra Manguoglu ${ }^{3}$, Guven Luleci ${ }^{3}$, Rebecca Doherty', Margaret Christie', Paul Craven', Elizabeth \\ Bancroft', Anita Mitra', Karolina Zieba', Ros Eeles' on behalf of the IMPACT steering committee and IMPACT collaborators
}

IInstitute of Cancer Research, London, United Kingdom; 2International Hereditary Cancer Centre, Pomeranian Medical University, Szczecin, Poland; ${ }^{3}$ Department of Medical Biology and Genetics, Faculty of Medicine, Akdeniz University, Antalya, Turkey

Submitted: 20 May 2006

Prostate cancer is a significant public health problem. In the European Union, approximately 200,000 men are diagnosed annually and the incidence is increasing, even allowing for the rise in screen-detected prostate cancer. Multiple aetiologies have been proposed to contribute to the development of prostate cancer. However, there is strong evidence that inherited genetic factors are important and contribute to significant familial aggregation in some men, particularly when affected at a young age (Woolf et al., 1960; Steinberg et al., 2000; Singh, 2000; Edwards et al., 2003). The breast cancer predisposition genes $B R C A 2$, and possibly also $B R C A 7$, have been reported to increase the risk of prostate cancer in male carriers of these genes; however, the prostate cancer risk associated with carriage of BRCA gene mutations remains uncertain.

IMPACT (Identification of Men with a genetic Predisposition to ProstAte Cancer: Targeted screening in BRCA1 and BRCA2 mutation carriers and controls) is the first multinational study to investigate screening and diagnosis for men with a genetic risk of prostate cancer. An international collaboration which currently involves over 20 countries, the study aims to identify and recruit 850 male BRCA1/BRCA2 mutation carriers, and an equal number of controls (men with a negative predictive BRCAl/2 genetic test), into a targeted screening programme for prostate cancer.

IMPACT aims to determine the sensitivity and specificity of PSA screening for prostate cancer in BRCAT/2 carriers and controls, and to evaluate new screening markers for this disease. It also aims to gain a better understanding of the pathogenicity of prostate cancer in men with $B R C A 7$ or BRCA2 mutations, and will attempt to identify new modifier genes or biomarkers for this population.

$B R C A 1$ and BRCA2 are the only genes, thus far, with an established role in increasing prostate cancer risk, but it has been suggested that a number of other genes exist which may predispose to prostate cancer (each accounting for a proportion of familial prostate cancer clusters). With the establishment of a solid IMPACT consortium, it is hoped this study could be extended to investigate other prostate cancer predisposition genes if and when they are identified.

Cancer research has been targeted as a high priority for the European Community (EC); however, research is most successful when centralised and well co-ordinated, avoiding the duplication and fragmentation associated with smaller, isolated studies. The EC Framework 6 Programme, which aims to facilitate co-operation between European countries in the field of cancer research, has therefore provided funding for IMPACT to be expanded to include clinical and research teams in Bulgaria, Romania and Turkey (and other Associated Candidate Countries and New Member States of the EU). A subteam of IMPACT, known as AIDIT (Advancing International Co-operation and Developing Infrastructure for Targeted Screening of Prostate Cancer in Men with Genetic Predisposition), will focus on linking researchers in these new countries with the established international IMPACT Consortium.

The EC's support of AIDIT provides the opportunity for teams who might be new to research in genetics or oncology to become integrated into an existing study. Communication between new and existing collaborators will allow the sharing of knowledge and formulation of strategies to overcome local barriers and challenges.

The AIDIT team is in the process of establishing a website to facilitate communication between current and potential project collaborators. It will also fund and facilitate an international conference to bring together research teams, clinicians and policy makers from across Europe. The project also aims to raise awareness 
Sarah Morgan, Jan Lubinski, Esra Manguoglu, Guven Luleci, Rebecca Doherty, Margaret Christie, Paul Craven, Elizabeth Bancroft, Anita Mitra, Karolina Zieba, Ros Eeles on behalf of the IMPACT steering committee and IMPACT collaborators

of familial prostate cancer among health professionals and the public within Associated Candidate Countries and New Member States of the EU.

Clinical and research teams who are interested in participating in IMPACT or who would like to attend the funded conference in Poland from 28 to 30 November are encouraged to contact the AIDIT administrator (email: impact-study@icr.ac.uk or telephone +44 (0) 208661 3897).

This publication reflects only the authors' views. The European Community is not liable for any use that may be made of the information contained herein. The information in this document is provided as is and no guarantee or warranty is given that the information is fit for any particular purpose. The user hereof uses the information at its sole risk and liability. 